\title{
Single port thoracoscopic treatment of thoracic duct injury after thyroidectomy with neck dissection
}

\author{
Serkan Teksoz ${ }^{1}$, Ezel Ersen ${ }^{2}$, Akif Enes Arikan ${ }^{1,3}$, Sina Ferahman ${ }^{1}$, Kamil Kaynak ${ }^{2}$, Gianlorenzo Dionigi ${ }^{4}$, \\ Yusuf Bukey $^{1}$ \\ ${ }^{1}$ Department of General Surgery, ${ }^{2}$ Department of Thoracic Surgery, Cerrahpasa Medical Faculty, Istanbul University, Istanbul, Turkey; ${ }^{3}$ Clinic of \\ General Surgery, Horasan State Hospital, Erzurum, Turkey; ${ }^{4}$ Division for Endocrine Surgery, Department of Human Pathology in Adulthood and \\ Childhood "G. Barresi”, the University of Messina, Messina, Italy \\ Correspondence to: Akif Enes Arikan. Cerrahpasa Tip Fakultesi, Genel Cerrahi AD 34098 Fatih, Istanbul, Turkey. Email: enesarikan@yahoo.com.
}

\begin{abstract}
Chylous leakage is a complication of thyroidectomy accompanied by bilateral neck dissection with incidence of $0.5-6.2 \%$. A 51-year-old female patient underwent total thyroidectomy, bilateral and central neck dissection for papillary thyroid carcinoma. In post-operative $4^{\text {th }}$ day, left sided chylous leakage was observed as $1,500 \mathrm{cc} /$ day through neck drain. Leakage did not cease after 1 -month conservative treatment so single port thoracoscopic intervention was performed. Under general anesthesia, patient was placed in left lateral decubitus position. An Alexis ${ }^{\circledR}$ retractor was placed through sixth intercostal space. Thoracic cavity was visualized with $30^{\circ}$ scope. Posteroinferior edge of lower lobe was retracted superior posteriorly with a Foerster clamp to display inferior pulmonary ligament, which was then divided with electrocautery. Posterior mediastinal pleura between azygous vein and chest wall was incised to mobilize the vein. After that, mediastinal pleura between azygous vein and esophagus was cut longitudinally and esophagus was retracted anteriorly to dissect towards aorta. By dissection, thoracic duct was revealed as a thin tubular structure with occasional peristalsis. After isolation of the duct, it was clipped using Hem-o-lok ${ }^{\circledR}$. Finally, fibrin sealant was applied to decrease risk of recurrence. One chest tube was placed to ensure adequate drainage of thoracic cavity and complete re-expansion of lung. Neck drain and chest tube was extracted in postoperative second and fourth day respectively and patient was discharged at $8^{\text {th }}$ day. Single port thoracoscopy is a safe choice for treatment of chylous leakages due to cervical ductus thoracicus injury with faster recovery.
\end{abstract}

Keywords: Thoracic duct; thoracoscopic approach; single port; thyroidectomy; neck dissection

Submitted Jun 25, 2017. Accepted for publication Jul 24, 2017.

doi: $10.21037 /$ gs.2017.07.14

View this article at: http://dx.doi.org/10.21037/gs.2017.07.14

\section{Introduction}

Cervical thoracic duct injuries are rare but are well-known complications with an incidence about $0.5-6.2 \%(1,2)$. Due to left side prominent termination of thoracic duct, these injuries occur predominantly on the left side of patients underwent to lateral neck dissection (1). Chylous fistulas occur between the first and third postoperative day in $86 \%$ of patients $(1,3)$.

In the literature, these injuries can be managed either conservatively or surgically $(1,4,5)$. Initial management of chylous fistula begins with dietary modifications such as diets poor in fat with medium chain triglycerides or total parenteral nutrition, repeated drainage, pressured dressings $(2,4)$. In addition, recent studies demonstrated that somatostatin and its analogues have high effectiveness in management of chylous fistulas, with less side effects $(2,3)$.

With recent developments in field of thoracoscopic surgery such as including endoscopic ligation of the ductus thoracicus, management of chest diseases are changed (4). However, by searching PubMed database with keywords "single", "thoracoscopy", and "duct" and Web of Knowledge 

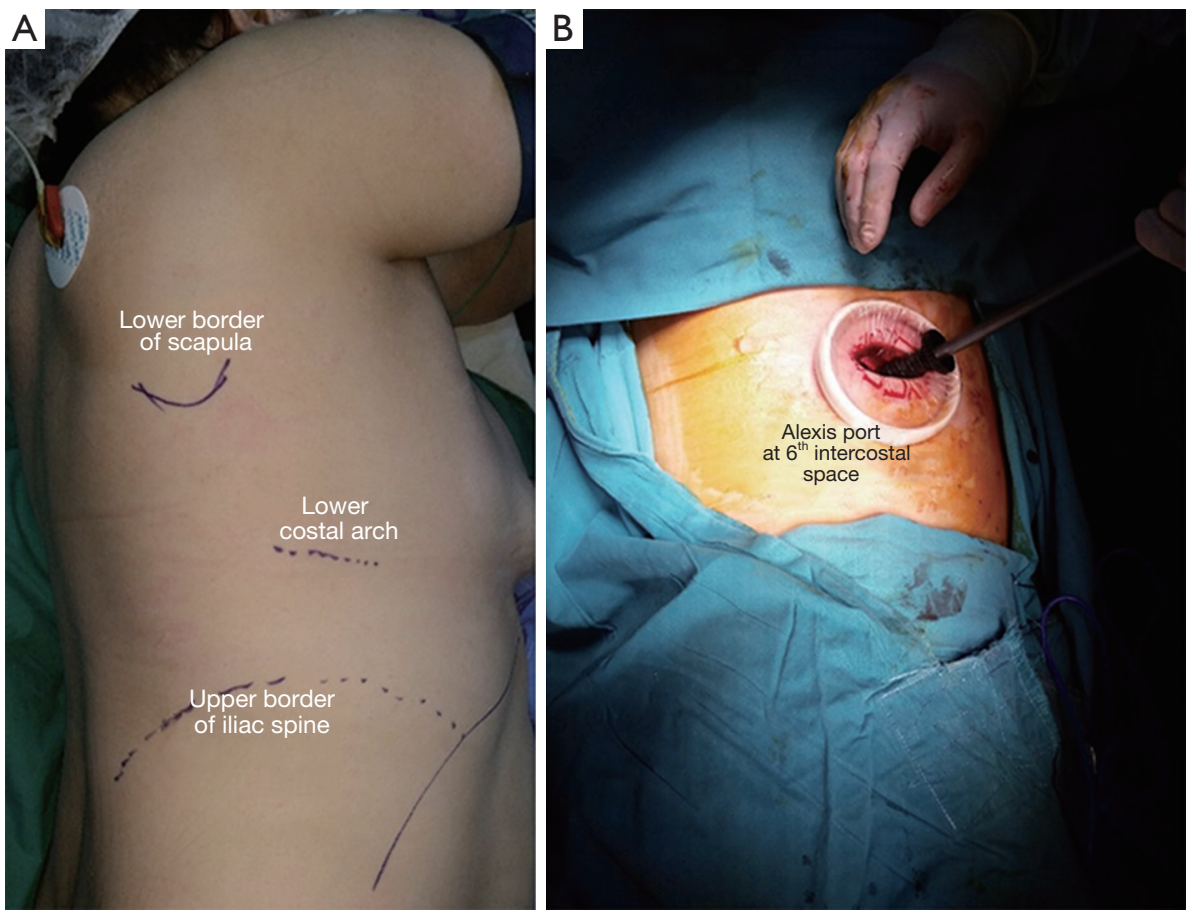

Figure 1 Position of the patient and location of port. (A) Position of the patient; (B) location of single port.

database with keywords "single", "thoracoscopy", and "duct" no report of single port thoracoscopic ligation of ductus thoracicus was found.

In this article, successful management of cervical chylous fistula following neck dissection due to advanced thyroid carcinoma by ligation of the ductus thoracicus via single port thoracoscopy is reported.

A written informed consent was obtained from the patient.

\section{Case presentation}

Fifty-one-year-old female, whose ultrasound showed bilateral lymph node metastases in addition to $12 \mathrm{~mm}$ primary tumor in right thyroid lobe was referred to our institution. Cytological examination of fine-needle aspiration biopsy specimen from the thyroid nodule and one of the right neck lymph node resulted as papillary thyroid carcinoma and metastasis. Total thyroidectomy accompanied by bilateral and central neck-dissection was performed to the patient. Before the operation was completed closed suction drain was inserted. Oral intake was started in postoperative $6^{\text {th }}$ h. Postoperative drainage was $650 \mathrm{~mL} /$ day. In post-operative $4^{\text {th }}$ day, left sided chylous leakage was observed as $1,500 \mathrm{cc} /$ day through neck drain. Oral intake was ceased, total parenteral nutrition containing medium chain triglycerides and somatostatin analogue $0.1 \mathrm{cc} 3 \times 1$ daily subcutaneous was started. Daily drainage volume was less than $600 \mathrm{~mL}$ at the $6^{\text {th }}$ day but the volume increased over $1,200 \mathrm{~mL} /$ day in $12^{\text {th }}$ day after operation. Leakage was not ceased after 1-month conservative treatment and patient underwent to single port thoracoscopic intervention.

Patient was positioned in left lateral decubitus under general anesthesia (Figure 1A). An Alexis-O-Wound Protector/Retractor (Applied Medical, Rancho Santa Margarita, CA, USA) was inserted through sixth intercostal space (Figure $1 B$ ). A $30^{\circ}$ scope was used to visualize chest. Inferior pulmonary ligament, which is divided after with electrocautery, visualized by superior posteriorly retraction of posteroinferior edge of lower lobe of the lung with a Foerster clamp. Posterior mediastinal pleura between azygous vein and chest wall was incised to mobilize the vein. After that, mediastinal pleura was cut longitudinally between azygous vein and esophagus thus esophagus was retracted anteriorly to dissect bluntly towards aorta. By blunt dissection, thoracic duct, a thin tubular structure presenting occasional peristalsis, was revealed. After isolation of the duct, it was clipped with Hem-o-lok ${ }^{\circledR}\left(\right.$ Weck $^{\circledR}$ 
Surgical Instruments, Teleflex Medical, Durham, NC, USA) clip. Finally, fibrin sealant was applied to decrease risk of recurrent chylothorax. One 32-Fr chest tube was remained to sustain adequate drainage of thoracic cavity and total re-expansion of lung. Performed operation can be seen in Figure 2. Neck drain and chest tube was extracted in postoperative second and fourth day respectively and patient was discharged at $8^{\text {th }}$ day. Wound healed with minimal scar (Figure $3 A, B$ ).

\section{Discussion}

Thoracic duct injury in neck dissection is rare but serious complication. During neck dissection, especially in level

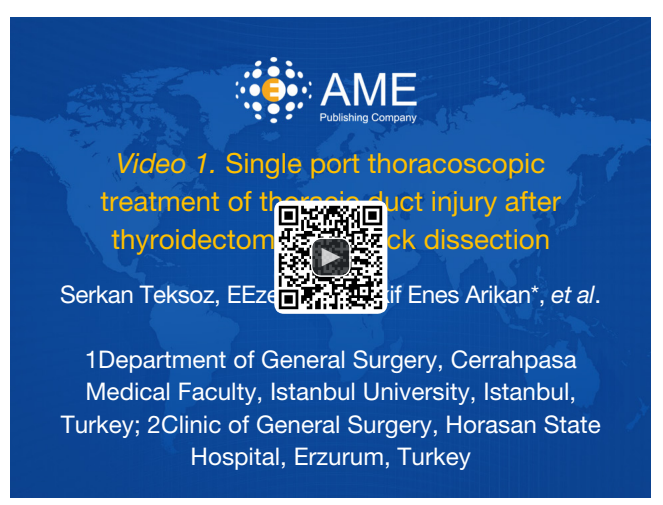

Figure 2 Single port thoracoscopic treatment of thoracic duct injury after thyroidectomy with neck dissection (6).

Available online: http://www.asvide.com/articles/1767
$\mathrm{IV}$, thoracic duct is vulnerable to damage because of high anatomic variation rate and its fragile structure hardens to repair. To prevent chylous fistulae looking for possible chyle leaks during surgery is crucial $(2,4)$. Recognition during surgery and over sewing or application of fibrin/ cyanoacrylate is a choice of treatment. If not recognized or intervened during surgery, use of a medium-chaintriglyceride or fat-free diet, total parenteral nutrition, somatostatin analogues, orlistat or etilefrine are options for conservative management (7).

However, no clear consensus for optimal management of the thoracic duct fistulas is existing. In current literature nutritional, surgical and pharmacological procedures have been proposed for treatment (8). In addition, there is still a doubt for choice of surgical or conservative treatment $(1,8,9)$.

When conservative treatment is not curative, reexploration is required. Unfortunately, surgical procedures in neck region for chylous leakage require suture ligation in numerous amount or a muscle bolster. However, ligation of ductus thoracicus in the thoracic cavity is the easiest and reliable procedure. Even though, ligation of ductus thoracicus can be performed by open thoracotomy technique, successful treatment of chyle fistula has been recently demonstrated with thoracoscopic approach. This procedure is effective and avoids the significant morbidity of major thoracic access $(3,10)$. Usually, right-sided approach is preferred for identification of ductus thoracicus. Ligation of tissue between the azygos vein and the aorta above supra-diaphragmatic hiatus sustains the occlusion of ductus thoracicus.
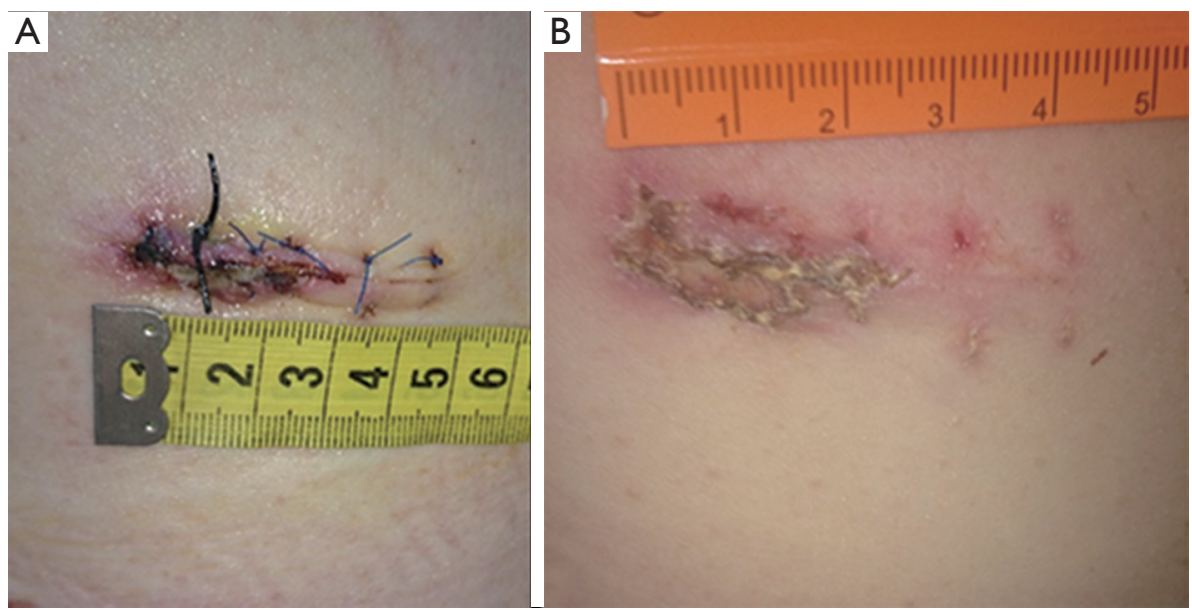

Figure 3 Wound healing. (A) Post-operative $4^{\text {th }}$ day (the day after removal of chest tube) $5 \mathrm{~cm}$ wound can be seen; (B) post-operative $10^{\text {th }}$ day cosmetically good wound healing. 


\section{Conclusions}

As reported in this case, safe and effective treatment of chylous fistula in the neck following neck dissection can be achieved by single port thoracoscopy. In addition to avoidance of morbidity associated with major thoracotomy, lesser pain and faster recovery is observed with single port thoracoscopic approach. Unnecessary and prolonged conservative treatment can be avoided in chylous fistula after neck dissection with single port thoracoscopic intervention in early phase.

\section{Acknowledgements}

None.

\section{Footnote}

Conflicts of Interest: The authors have no conflicts of interest to declare.

Informed Consent: Written informed consent was obtained from the patient for publication of this manuscript and any accompanying images.

\section{References}

1. Rodier JF, Volkmar PP, Bodin F, et al. Thoracic duct fistula after thyroid cancer surgery: towards a new treatment? Case Rep Oncol 2011;4:255-9.

Cite this article as: Teksoz S, Ersen E, Arikan AE, Ferahman S, Kaynak K, Dionigi G, Bukey Y. Single port thoracoscopic treatment of thoracic duct injury after thyroidectomy with neck dissection. Gland Surg 2017;6(5):598-601. doi: 10.21037/ gs.2017.07.14
2. Süslü N, Sözeri B, Hoşal AS, et al. The Role of Somatostatin Treatment in the Management of Chylous Fistula after Neck Dissection. Turk Arch Otolaryngol 2014;52:39-42.

3. Lorenz K, Abuazab M, Sekulla C, et al. Management of lymph fistulas in thyroid surgery. Langenbecks Arch Surg 2010;395:911-7.

4. Campisi CC, Boccardo F, Piazza C, et al. Evolution of chylous fistula management after neck dissection. Curr Opin Otolaryngol Head Neck Surg 2013;21:150-6.

5. Ikeda Y. Thoracoscopic management of cervical thoracic duct injuries after thyroidectomy with lymphadenectomy. Asian J Endosc Surg 2014;7:82-4.

6. Teksoz S, Ersen E, Arikan AE, et al. Single port thoracoscopic treatment of thoracic duct injury after thyroidectomy with neck dissection. Asvide 2017;4:451. Available online: http://www.asvide.com/articles/1767

7. Brennan PA, Blythe JN, Herd MK, et al. The contemporary management of chyle leak following cervical thoracic duct damage. Br J Oral Maxillofac Surg 2012;50:197-201.

8. Lee YS, Nam KH, Chung WY, et al. Postoperative complications of thyroid cancer in a single center experience. J Korean Med Sci 2010;25:541-5.

9. Roh JL, Kim DH, Park CI. Prospective identification of chyle leakage in patients undergoing lateral neck dissection for metastatic thyroid cancer. Ann Surg Oncol 2008;15:424-9.

10. Zoetmulder F, Rutgers E, Baas P. Thoracoscopic ligation of a thoracic duct leakage. Chest 1994;106:1233-4. 